\title{
Automatic Blood Vessel Extraction of Fundus Images Employing Fuzzy Approach
}

\author{
Charu Bhardwaj ${ }^{1}$, Shruti Jain ${ }^{2}$, Meenakshi Sood ${ }^{3}$ \\ 1,2,3 Department of Electronics and Communication Engineering, JUIT Waknaghat, Solan, India
}

\begin{tabular}{l} 
Article Info \\
\hline Article history: \\
Received Jan 28, 2019 \\
Revised May 1, 2019 \\
Accepted Nov 4, 2019 \\
\hline Keyword: \\
Blood Vessel Extraction \\
Diabetic Retinopathy \\
Exudates \\
Fuzzy \\
Hemorrhages \\
Kirsch's Algorithm.
\end{tabular}

Charu Bhardwaj,

Department of Electronics and Communication Engineering,

JUIT Waknaghat, Solan, India

Email: cbcharubhardwaj215@gmail.com

\begin{abstract}
Diabetic Retinopathy is a retinal vascular disease that is characterized by progressive deterioration of blood vessels in the retina and is distinguished by the appearance of different types of clinical lesions like microaneurysms, hemorrhages, exudates etc. Automated detection of the lesions plays significant role for early diagnosis by enabling medication for the treatment of severe eye diseases preventing visual loss. Extraction of blood vessels can facilitate ophthalmic services by automating computer aided screening of fundus images. This paper presents blood vessel extraction algorithms with ensemble of pre-processing and post-processing steps which enhance the image quality for better analysis of retinal images for automated detection. Extensive performance-based evaluation of the proposed approaches is done over four databases on the basis of statistical parameters. Comparison of both blood vessel extraction techniques on different databases reveals that fuzzy based approach gives better results as compared to Kirsch's based algorithm. The results obtained from this study reveal that $89 \%$ average accuracy is offered by the proposed MBVEKA and $98 \%$ for proposed BVEFA.
\end{abstract}

Copyright $\left({ }_{0} 2018\right.$ Institute of Advanced Engineering and Science. All rights reserved.

\section{INTRODUCTION}

Diabetes accounts in majority of eye disease and Diabetic Retinopathy (DR) is the most severe complications resulting from prolonged diabetic condition. DR is a condition arising from high glucose level and causes damage to the small blood vessels inside the retina. These tiny blood vessels leak blood and fluid into the retina, forming lesions such as microaneurysms, hemorrhages, hard exudates, cotton wool spots, etc. DR is a severe eye disease which may cause serious visual impairments and even causes blindness if left untreated [1]. According to the World Health Organization (WHO), typically more than $75 \%$ of patients having diabetes mellitus for a period of more than 20 years of age may develop certain amount of DR [2,3]. Nonproliferative Diabetic Retinopathy (NPDR) indicates the first clinical symptom of DR and this stage is the consequence of direct damage to the retinal blood vessels. In this condition, the damaged blood vessels leak some amount of fluid or blood into the retina [4]. There are large number of cases of DR patients, thus DR is likely to pose a threat to clinical fraternities. To facilitate ophthalmologists, processing of fundus images to develop Computer Aided Diagnostic (CAD) systems play a vital role. It poses the need of blood vessel extraction for further processing of fundus images to be classified for the treatment of serious eye issues like diabetic retinopathy.

Many researchers have confronted this challenging job of extracting blood vessels through various novel techniques still this is a daunting challenge and requires more work on finding a novel technique for efficient blood vessel extraction. Authors in [5] have proposed a Curvelet based Edge Enhancement technique to detect the edges of dark lesions from the background. For statistical performance analysis, receiver operating characteristics were evaluated and it was revealed that the proposed method is slightly weaker for red lesion detection as they are indistinguishable from background. In [6] the authors have proposed an approach of 
setting candidate MAs using simple threshold from the pre-processed image and then selecting the clutter among the candidates by using rejectors in cascaded form. Morphological and appearance-based features are used to characterize the clutter and MA structures. A novel detection approach for red lesion detection was introduced in [7] based on a new set of dynamic shape features which was evaluated in six different databases for the detection of MAs and HEMs. The method outperforms many state-of-the-art approaches due to its robust features and high discrimination capabilities at both per-lesion and per-image levels. A method was presented in [8] to detect MAs by analysing directional cross-section profiles which is centred in the candidate pixel of the pre-processed image. A new template- based approach was presented in [9] for optical disc segmentation using a circular optical disc boundary approximation which offers good compromise between success rate, quality and efficiency. Authors in [3] proposed a Probabilistic Fuzzy C-mean technique and classification was done using neuro-fuzzy classifiers to provide highest accuracy among other state-of-art techniques. Authors in [10] employed a fuzzy image processing approach to provide a reliable screening system for early detection of sight threatening diseases. In [11], to evaluate the vascular structure for DR canny edge detector is used. This detector helps to produce continuous edges as well as better image quality for identifying blockages in tiny blood vessels. Authors in [12] employs pre-processing and feature extraction techniques in which features extracted for the study are specifically chosen due to their biological relevance and previously reported results for early detection of DR. A novel dataset was introduced in [13] useful for researchers working in the retinal imaging area to produce more reliable screening system. OSTU thresholding based segmentation strategy was proposed in [14] and SVM classifiers are used for classification. This algorithm achieves achieve a mean sensitivity of $95.05 \%$ and a mean positive predictive value of $95.37 \%$. A morphological method was proposed in [15] and thresholding is also used to detect the clinical symptoms faster and efficiently so as to facilitate the ophthalmologists during exudates screening process. A Graphical User Interface (GUI) was given in [16] Kirsch's template for the accurate detection of Exudates. This GUI is helpful in incorporating maximum number of image processing techniques altogether to obtain higher performance. Exceptional performance was achieved in [17] for blood vessels and optic disk segmentation in retinal images by employing Markov Random Field (MRF) image reconstruction method. Authors in [18] detected the clinical features by using thresholding function and morphological operations so as to reveal retinal abnormalities. Several image processing techniques were evaluated in [19] for detecting lesions and it was concluded that the chances for vision loss can be reduced upto $50 \%$ by early diagnosis and screening of DR.

From diagnostic point of view, analysis of retinal image is getting more and more important nowadays. In automatic image analysis, Optic Disc (OD), optic cup, and blood vessels are the most essential anatomic landmarks to extract. Though the researchers have come up with various novel techniques for OD removal still there is a lot of scope for efficient blood vessel extraction. Henceforth, authors in this paper proposed two blood vessel extraction techniques; modification of Blood Vessel Extraction using Kirsch's Approach (MBVEKA) and Blood Vessel Extraction using Fuzzy Approach (BVEFA). Both the techniques involve pre-processing and post processing steps that exhibit improved diagnostic performance. Blood vessel extraction techniques employed for this research work can facilitate ophthalmic services by automated computer aided screening of fundus images. Early detection of DR may prevent visual loss by enabling medication or laser therapy for the treatment of severe eye diseases. Critical performance analysis of the proposed algorithms are validated qualitatively and quantitatively.

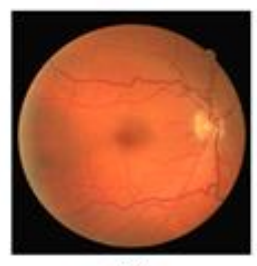

(a)

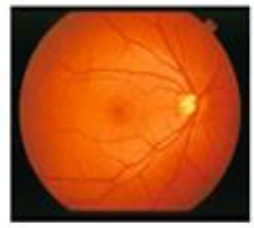

(c)

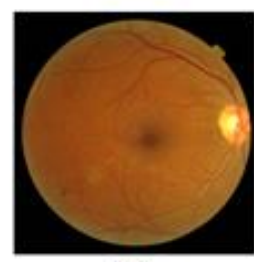

(b)

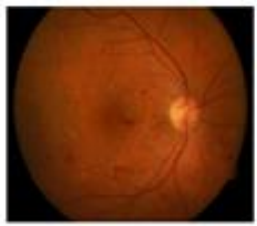

(d)

Figure 1. Fundus images taken from different databases (a) Fundus image from DRIVE database (b) Fundus image from MESSIDOR database (c) Fundus image from STARE database (d) Fundus image from DIARETDB1 database 


\section{MATERIAL AND METHODS}

\subsection{Fundus Image Databases}

To generalize our research work and validate the proposed techniques, various publically available standard retinal databases DRIVE [20], STARE [21], DIARETDB1 [22] and MESSIDOR [23] are explored for image analysis. These databases are used for image testing, blood vessels extraction and segmentation [24], feature extraction [25] and classification for DR screening and detection[26]. Fundus images acquired from different databases are shown in Figure 1 and they are detailed in the subsequent sections.

\subsection{Proposed Methodology}

The reasons for image artefacts altering image analysis are poor focus of camera, movement of patient, reflections, bad positioning of lens, inappropriate illumination, etc [3]. To remove these image artefacts, image pre-processing steps are followed to mitigate or even eliminate these problems, prior to applying the localization of OD and extraction of blood vessels. This paper proposes MM based OD detection technique for accurate optical disc segmentation and removal followed by MBVEKA and BVEFA approaches for blood vessel extraction. Blood vessel extraction techniques employed enhances noise too, so post processing techniques are employed for their removal. The proposed algorithms are employed on a large number of images taken from 4 standard datasets listed in section 2.1 but due to space contrastaints the results of ten images from every dataset are reported in this research paper. Performance evaluation is done for both the proposed techniques employing various performance metrics to validate the goodness of prposed algorithms. The block diagram of the proposed Blood Vessel Extraction Techniques is shown in Figure 2.

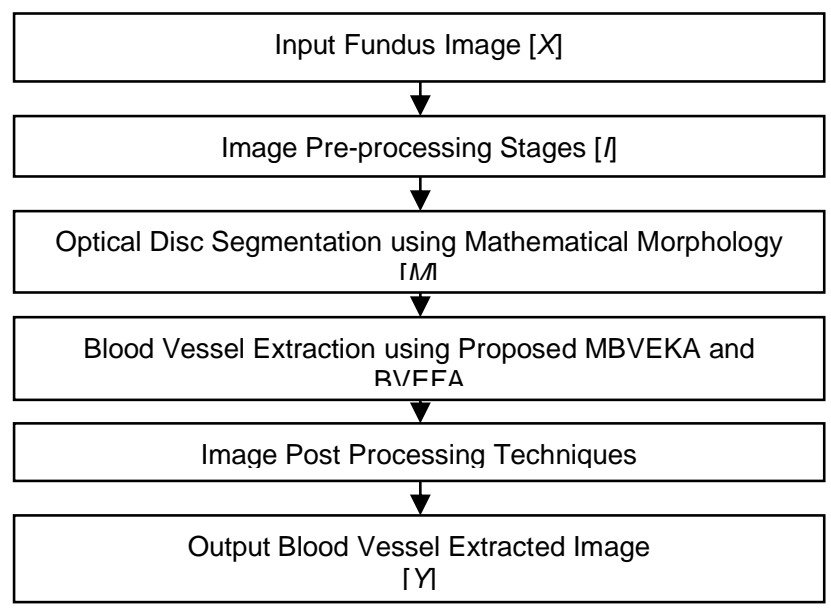

Figure 2. Block Diagram of Proposed Blood Vessel Extraction Techniques

\subsubsection{Image Variations Attenuation}

Different individuals have different iris color and skin pigmentation of individuals is different causing variations in the fundus image captured from retinas. These artefacts are significant enough to hinder the human grading for abnormality detection. So, the fundus image is normalized to make it invariant with respect to the background pigmentation variation between different individuals. In this research work, image variation attenuation is done using Grayworld normalization [27]. In this method, change in illumination is modelled by three constant scaling factors $\alpha, \beta$ and $\gamma$ in $\mathrm{R}, \mathrm{G}$ and $\mathrm{B}$ color channels respectively and averaging the value by;

$$
(\alpha \mathrm{R}, \beta \mathrm{G}, \gamma \mathrm{B})=\left(\frac{\alpha \mathrm{R}}{\frac{\alpha}{n} \sum_{\mathrm{i}} \mathrm{R}}, \frac{\beta \mathrm{G}}{\frac{\beta}{n} \sum_{\mathrm{i}} \mathrm{G}}, \frac{\gamma \mathrm{B}}{\frac{\gamma}{n} \sum_{\mathrm{i}} \mathrm{B}}\right)
$$

RGB to grayscale conversion is an important step in image pre-processing and is done by;

$$
\mathrm{X}_{\mathrm{g}}=0.33 * \mathrm{R}+0.5 * \mathrm{G}+0.166 * \mathrm{~B}
$$

where the output gray image is $I$ and the red, green, and blue components are $R, \mathrm{G}$, and $B$ respectively.

\subsubsection{Intensity Conversion}

The presence of three channels, red, green and blue in every image leads to another important step of intensity conversion. Red channel cannot be used for processing as it is of same color as that of the blood vessels, blue channel is very dark, possessing noise component and has low contrast. Thus for intensity conversion green channel is used as it has the maximum intensity and all the features in fundus image are clearly visible in green channel [28]. 


\subsubsection{Median Filtering}

Image acquisition using CCD cameras, sensor temperature and light illuminated are the major factors affecting the noise in the acquired image requiring denoising. Median filter is used in our research work to suppress the isolated noise without blurring the edges. Averaging filter is also a good choice to suppress the isolated noise but this in turn blurs sudden changes like sharp edges, discontinuities and some other image details which may be important for analysis purpose. Median filter is used which replaces a pixel in the image by the median value, instead of average value of pixels in the neighbourhood. Thus median filter is the appropriate choice to distinguish isolated noise from legitimate image features like some kind of discontinuities, edges or lines. Expression for median filter is given by;

$$
\mathrm{I}^{\prime}(\mathrm{m}, \mathrm{n})=\operatorname{median}\{\mathrm{I}[\mathrm{i}, \mathrm{j}]\} \quad,(\mathrm{i}, \mathrm{j}) \in \mathrm{w}
$$

where, $I[i, j]$ is the original image and after applying median filtering image is converted to $\mathrm{I}^{\prime}$. $\mathrm{w}$ represents the neighbourhood centred around location [m, n] in the image [29].

\subsubsection{Contrast Enhancement}

The contrast of the fundus image is maximum at the centre of the image and it decreases rapidly while moving in the outward direction from the centre of the image. For local contrast enhancement, histogram equalization method is used, however, when image contrast characteristics vary across the image then this method becomes less effective. An improvement made is Adaptive Histogram Equalization (AHE) which is used for contrast varying characteristics of the image but it in turn amplifies the noise in the image. Another modification in histogram equalization is Contrast Limited Adaptive Histogram Equalization (CLAHE) which gives effective contrast enhancement and even overcome the drawbacks of AHE [29].

For this research work CLAHE is used as it operates on small data regions rather than the entire image and the contrast of each region is enhanced so that the histogram of the output region approximately matches the specific histogram distribution [28]. Let RGB be the original fundus image represented by a matrix of integer pixel intensities ranging from a dynamic range of 0 to $\mathrm{L}-1$. L is the maximum value of intensity values i.e. 256. The normalized histogram of the original image is denoted by $\mathrm{pn}$,

$$
\begin{aligned}
& \mathrm{p}_{\mathrm{n}}=\frac{\text { number of pixels with intensity } \mathrm{n}}{\text { Total number of pixels }} \\
& \text { for } \mathrm{n}=0,1, \ldots \ldots \ldots . . \mathrm{L}-1
\end{aligned}
$$

Thus the histogram equalized image is given by

$\mathrm{f}_{\mathrm{i}, \mathrm{j}}=$ floor $\left((\mathrm{L}-1) \sum_{\mathrm{n}=0}^{\mathrm{f}_{\mathrm{i}, \mathrm{j}}} \mathrm{p}_{\mathrm{n}}\right)$

where floor function rounds off to the nearest integer value.

\subsubsection{Optical Disk Segmentation using Morphological Approach}

Optical disc is an important feature to be eliminated for blood vessel extraction and in this research work morphological operations are employed [30]. The two main morphological operations are dilation and erosion, and the other two are opening and closing. In this research work after determining the centroid of the image, optical disc boundaries are detected based on morphological operations. The algorithm designed for OD segmentation using MM approach is given below.

\section{Algorithm 1: Mathematical Morphology for Optical Disc Segmentation \\ Input: Input Image $I=\mathrm{f}(\mathrm{x}, \mathrm{y})$ \\ Output: Output Image after removal of optical disc $[M]$}

\section{BEGIN}

Step 1: Extract the Green channel image and complement it.

Step 2: Apply adaptive histogram equalization on the complemented image.

Step 3: Apply morphological opening operation.

Erosion of image $\mathrm{A}$ and structuring element $\mathrm{B}$ is given by;

$A \ominus B=\left\{z \mid(B)_{z} \cap A^{C} \neq \varphi\right\}$ Set of all points z such that the intersection of $(B)_{z}$ with A's complement.

Dilation of image $\mathrm{A}$ with structuring element $\mathrm{B}$ is given by;

$A \oplus B=\left\{z \mid(\widehat{B})_{z} \cap A \neq \varphi\right\}$ Set of all points $z$ such that the intersection of $(\widehat{B})_{z}$ with A.

Opening operation is given by;

$$
A \circ B=(A \ominus B) \oplus B
$$

Step 4: Subtract the Morphologically opened image from the adaptive histogram equalized image for the removal of optical disc.

END

IJEEI, Vol. 7, No. 4, Dec 2019: 757 - 771 


\subsubsection{Edge Detection for Blood Vessel Extraction}

Edge detection is used to identify the points in the digital image at which discontinuities occur and the image brightness changes sharply across the image. Edge detection produces small intensity difference between two neighbourhood pixels which represents an edge [29]. For edge detection, Kirsch's algorithm and fuzzy approach are proposed in this research work.

\section{Modified Blood Vessel Extraction using Kirsch's Approach (MBVEKA):}

In the proposed MBVEKA algorithm, Kirsch's template is used for blood vessel extraction from fundus images and Kirsch's template is rotated at an angle of $45^{\circ}$ anticlockwise in all the 8 compass directions for edge enhancement and detection. The convolution is performed for each pixel of the image and eight template impulse responses to obtain image gradients in different directions. Finally all the gradients are summed up considering the directions for all of the RGB channels [31]. The Kirsch's template is shown in Figure 3.

\begin{tabular}{|c|c|c|}
\hline 5 & -3 & -3 \\
\hline 5 & 0 & -3 \\
\hline 5 & -3 & -3 \\
\hline \multicolumn{3}{|c|}{$0^{\circ}$}
\end{tabular}

\begin{tabular}{|c|c|c|}
\hline-3 & -3 & 5 \\
\hline-3 & 0 & 5 \\
\hline-3 & -3 & 5 \\
\hline \multicolumn{3}{|c|}{$180^{\circ}$} \\
\hline
\end{tabular}

\begin{tabular}{|c|c|c|}
\hline-3 & -3 & -3 \\
\hline-3 & 0 & -3 \\
\hline 5 & 5 & 5 \\
\hline \multicolumn{3}{|c}{$90^{\circ}$} \\
\hline
\end{tabular}

\begin{tabular}{|c|c|c|}
\hline 5 & 5 & 5 \\
\hline-3 & 0 & -3 \\
\hline-3 & -3 & -3 \\
\hline \multicolumn{3}{|c|}{$270^{\circ}$} \\
\hline
\end{tabular}

$270^{\circ}$

\begin{tabular}{|c|c|c|}
\hline-3 & -3 & -3 \\
\hline 5 & 0 & -3 \\
\hline 5 & 5 & -3 \\
\hline \multicolumn{3}{|c|}{$45^{\circ}$}
\end{tabular}

\begin{tabular}{|c|c|c|}
\hline-3 & 5 & 5 \\
\hline-3 & 0 & 5 \\
\hline-3 & -3 & -3 \\
\hline \multicolumn{3}{|c|}{$225^{\circ}$}
\end{tabular}

\begin{tabular}{|c|c|c|}
\hline-3 & -3 & -3 \\
\hline-3 & 0 & 5 \\
\hline-3 & 5 & 5 \\
\hline
\end{tabular}

\begin{tabular}{|c|c|c|}
\hline 5 & 5 & -3 \\
\hline 5 & 0 & -3 \\
\hline-3 & -3 & -3 \\
\hline \multicolumn{3}{|c|}{$315^{\circ}$}
\end{tabular}

Figure 3. Kirsch's Template

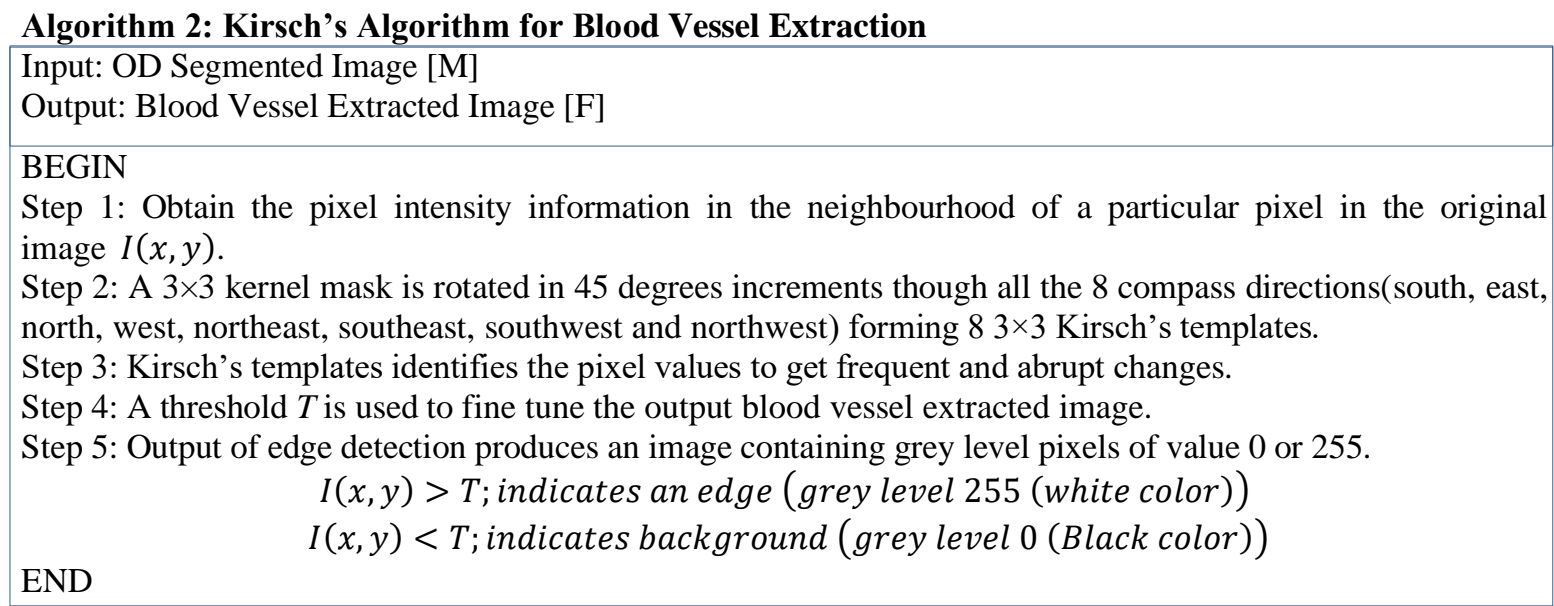

\section{Blood Vessel Extraction using Fuzzy Approach (BVEFA):}

Edge detection is the most important task for blood vessel extraction. The blood vessels are indicated as the edges or boundaries which may abruptly change with respect to the image gray level, Fuzzy approach is based on if-then rules unlike classical edge detection techniques becoming preference for edge detection. Classical edge detection techniques are good for fixed edge thickness applications but blood vessels have varying thickness across the retinal area which can be better addressed using fuzzy logic which provides a framework to contend with the uncertain information. Fuzzy approach provides the flexibility of adapting blood vessel thickness parameters easily either by adding a new fuzzy rule or by changing the output parameters [32].

In BVEFA technique, OD removed image is normalized using a scaling factor and then gradients in $\mathrm{x}$ and $\mathrm{y}$ direction are determined. Fuzzy inference is drawn and the membership functions are specified. Gaussian membership function is utilized in this algorithm due to its symmetric nature. Edges in the image are obtained by parsing fuzzy rule. The algorithm for the proposed BVEFA approach is depicted in the algorithm below. 


\section{Algorithm 3: Fuzzy Algorithm for Blood Vessel Extraction}

Input: OD Segmented Image [M]

Output: Blood Vessel Extracted image $[F]$

\section{BEGIN}

Step 1: Take the input image and normalize it using a scaling factor.

Step 2: Obtain image gradients in $x$ and $y$ direction by applying convolution.

Step3: Define a fuzzy inference system for edge detection and also specify its membership function.

Step 4: Evaluate the output of the edge detector for each row of pixels in $I(x, y)$ using corresponding rows of $\mathrm{I}_{\mathrm{x}}$ image gradient in $x$ direction and $I_{y}$ image gradient in $y$ direction specified as follows;

$$
I(x, y)=\text { white for } I_{x}=0 \text { and } I_{y}=0 \text {; indicating the background }
$$

END

$$
I(x, y)=\text { black for } I_{x} \neq 0 \text { and } I_{y} \neq 0 \text {; indicating an edge }
$$

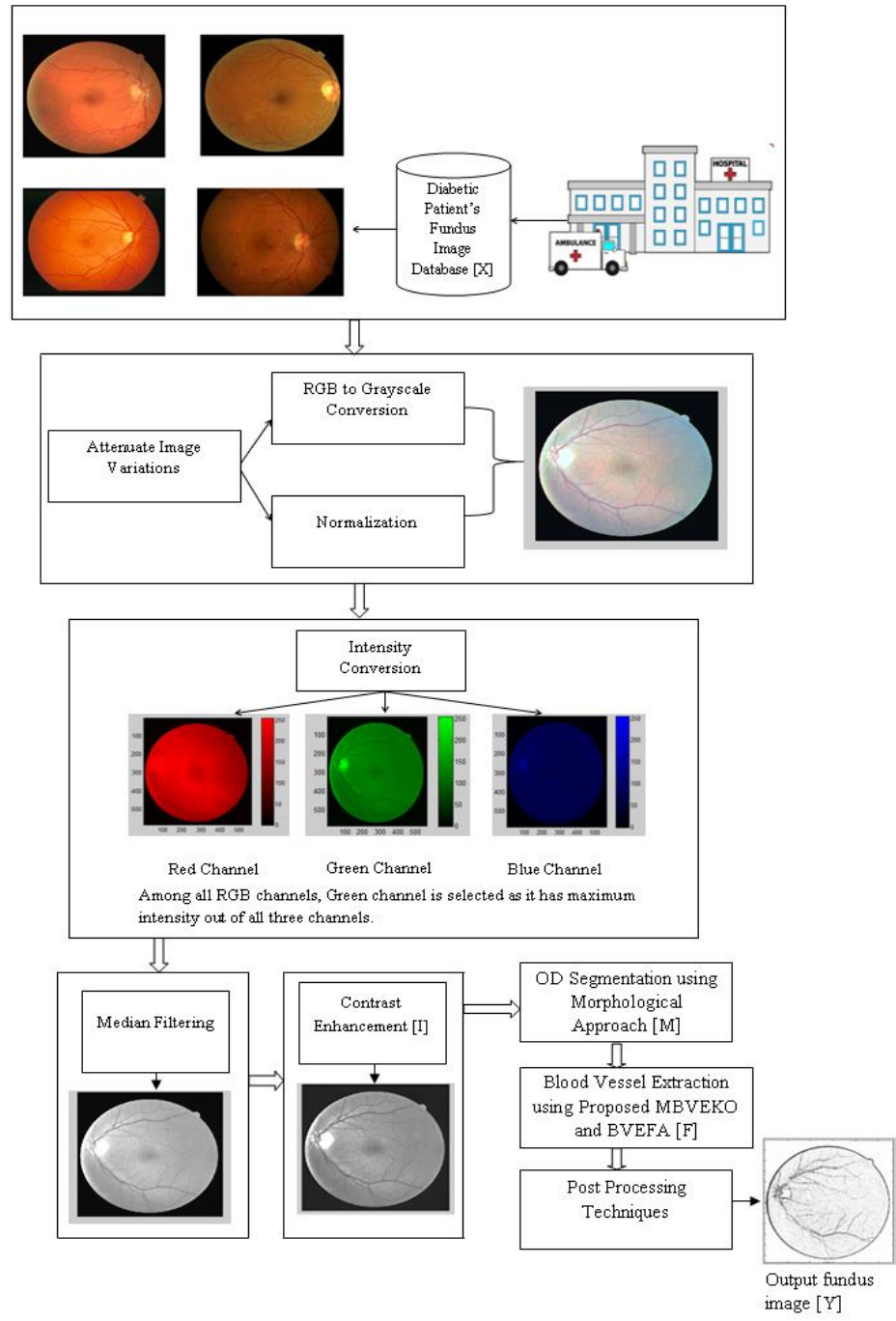

Figure 4. Flowchart of Proposed Blood Vessel Extraction Technique

\subsubsection{Post-processing}

Post-processing is the last step in the proposed approach and it is essential for efficient blood vessel extraction. This is done so as to overcome one of the drawbacks of using Kirsch's operator that it thickens the blood vessels more than their actual width and noise is enhanced using MBVEKA technique. Presence of noise may lead to misclassification of blood vessel as it can hamper the exact vessel pixel location with the isolated pixels formed due to noise. Thus morphological operations are used to thin the blood vessels along with median filtering to remove salt and pepper noise presents in fundus images after edge detection. Post processing steps involved in the proposed algorithm also removes the isolated pixels which are not considered as the part of the blood vessels [31]. The flowchart of proposed blood vessel extraction technique is given in Figure 4. 


\subsection{Performance Evaluation Metrics}

Various performance evaluation parameters are used in this research work for assessment of proposed blood vessel extraction techniques. These parameters are indicative of image quality obtained after applying the proposed algorithms.

\subsubsection{Jaccard Index}

This is the statistical measure of similarity between the two sets and defined as the size of intersection divided by that of the size of union of the two sample images taken for consideration.

Let $\mathrm{A}$ and $\mathrm{B}$ are the two images and $\mathrm{A}$ denotes the original gold standard image and $\mathrm{B}$ denotes the extracted image after image processing then Jaccard index is given by;

$$
J(A, B)=\frac{|A \cap B|}{|A \cup B|}
$$

\subsubsection{Dice Coefficient}

This is also a statistical measure for comparing the similarity between the two sample images.

Let $\mathrm{A}$ and $\mathrm{B}$ are the two images and $\mathrm{A}$ denotes the original gold standard image and $\mathrm{B}$ denotes the extracted image after image processing then the Dice coefficient is given by the formula;

$$
\mathrm{D}(\mathrm{A}, \mathrm{B})=\frac{2 \times|\mathrm{A} \cap \mathrm{B}|}{|\mathrm{A}|+|\mathrm{B}|}
$$

\subsubsection{SSIM (Structural Similarity Index) and DSIM (Structural Dissimilarity Index)}

SSIM is the similarity index based on statistical values of image or video attributes like brightness, texture, contrast, luminance and orientation. Its value lies between 0 and 1 and the pre-processed image is structurally similar to the original image if it approaches 1 .

SSIM between original image $\mathrm{x}$ and pre-processed image $\mathrm{y}$ is given by,

$\operatorname{SSIM}(\mathrm{x}, \mathrm{y})=\frac{\left(2 \mu_{\mathrm{x}} \mu_{\mathrm{y}}+\mathrm{c}_{1}\right)\left(2 \sigma_{\mathrm{xy}}+\mathrm{c}_{2}\right)}{\left(\mu_{\mathrm{x}}^{2}+\mu_{\mathrm{y}}^{2}+\mathrm{c}_{1}\right)\left(\sigma_{\mathrm{x}}^{2}+\sigma_{\mathrm{y}}^{2}+\mathrm{c}_{2}\right)}$

where $\mu_{\mathrm{x}}, \mu_{\mathrm{y}}$ denotes mean value of original image $\mathrm{x}$ and the pre-processed image y respectively, $\sigma_{\mathrm{x}}, \sigma_{\mathrm{y}}$ denotes variance value of $\mathrm{x}$ and $\mathrm{y}$ respectively. $\sigma_{\mathrm{xy}}$ is the covariance between $\mathrm{x}$ and $\mathrm{y}$ and $\mathrm{c}_{1}$ and $\mathrm{c}_{2}$ are two variables to stabilize the division with weak denominator.

Dissimilarity index giving the similarity variation of pre-processed image as compared to the original one is given by;

$$
\operatorname{DSIM}=\frac{1-\operatorname{SSIM}(\mathrm{x}, \mathrm{y})}{2}
$$

\subsubsection{Sensitivity, Specificity and Accuracy}

Sensitivity is defined as the statistical measure of proportion of correctly identified positives. Specificity is the measure of proportion of correctly identified negatives. Accuracy refers to the closeness of the measured value of result to that of the true value [33].

The formulas for Sensitivity, Specificity and Accuracy are given by;

$$
\begin{aligned}
& \text { Sensitivity }=\frac{\text { True Positive }}{\text { True Positive+False Negative }} \\
& \text { Specificity }=\frac{\text { True Negative }}{\text { True Negative+FalsePositive }} \\
& \text { Accuracy }=\frac{\text { True Positive+True Negative }}{\text { True Positive+False Negative+True Negative+FalsePositive }}
\end{aligned}
$$

\section{RESULTS AND DISCUSSION}

The results of both the proposed algorithms implemented on DRIVE database and performance analysis done for the fundus images are depicted in the following section using MATLAB2018 software. Various experiments performed with MBVEKA and BVEFA are detailed in this section considering all the databases listed in section 2.1. The performance metrics: Root Mean Square Error (RMSE), Peak Signal-to-Noise Ratio (PSNR), SSIM (Structural Similarity Index) and run time elapsed are evaluated to validate the algorithm [34,35].

Figure 5 shows the various stages for blood vessels extraction using MBVEKA which includes original image normalization to remove inter image variability, green channel conversions to obtain maximum contrast, denoising, CLAHE followed by OD removal and blood vessel extraction steps followed by post-processing. 
Figure $5(\mathrm{~g})$ depicts MBVEKA output which exhibit certain gaps and enhances the unwanted noise due to mismatching of Kirsch's template indicated by small dots in the output image. To overcome this limitation of MBVEKA and provide clear blood vessel distinction from the retinal background, BVEFA has been proposed and its results are depicted in Figure 6.

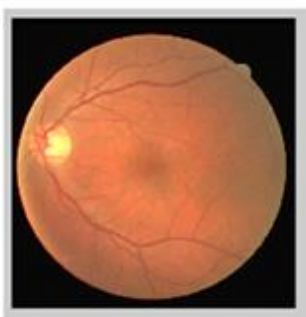

(a)

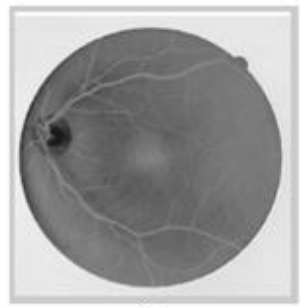

(e)

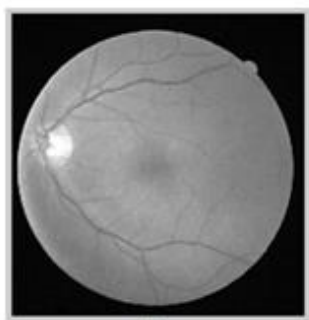

(b)

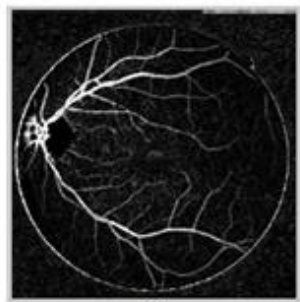

(f)

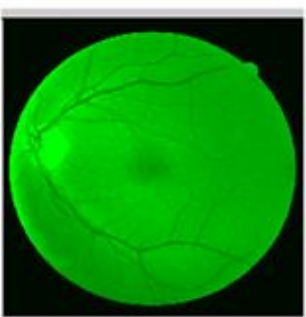

(c)

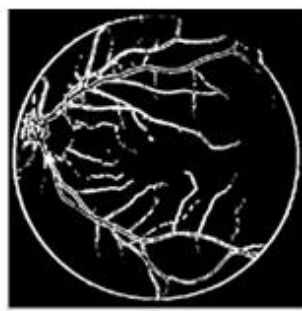

(g)

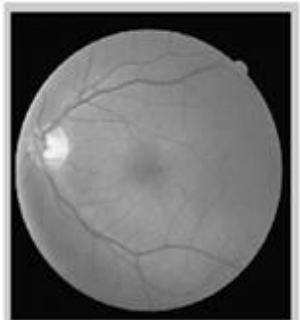

(d)

Figure 5. Modified Blood Vessel Extraction using Kirsch's Approach (MBVEKA) (a) Input fundus image (b) Variation attenuated image (c) Green channel extracted image (d) Median filtered image (e) CLAHE image

(f) Blood Vessels after OD removal (g) Extracted blood vessels using MBVEKA Algorithm

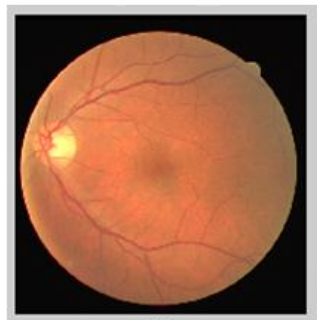

(a)

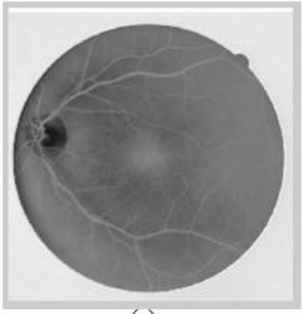

(e)

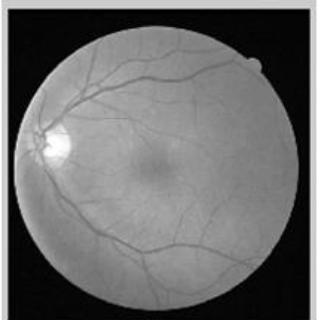

(b)

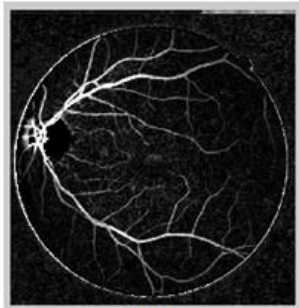

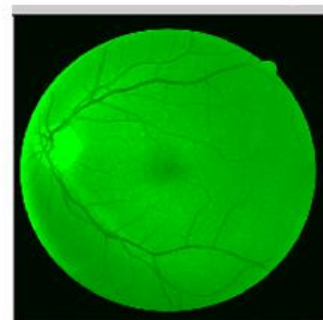

(c)

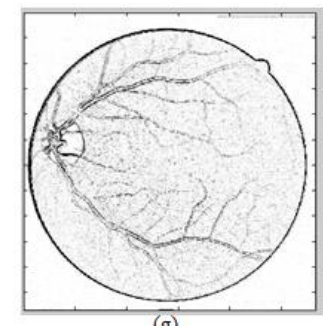

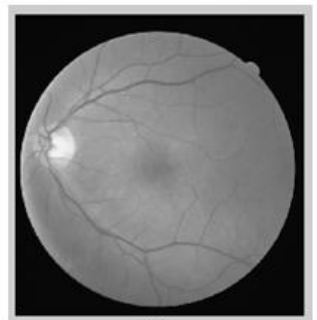

(d)

Figure 6. Blood Vessel Extraction using Fuzzy Approach (BVEFA) (a) Input fundus image (b) Variation attenuated image (c) Green channel extracted image (d) Median filtered image (e) CLAHE image (f) Blood Vessels after OD removal (g) Extracted blood vessels using BVEFA Algorithm

Figure 6 depicts the proposed BVEFA technique for blood vessel extraction. The stages include image variation attenuation to make image invariant to background illumination, green channel extraction, denoising, CLAHE equalization followed by optical disc removal and blood vessel extraction using BVEFA approach followed by post processing.From the visual representation of two proposed techniques it can be seen that blood vessels are better extracted using the fuzzy approach than that of the simplified blood vessel extraction approach employing Kirsch's operator. The visual results are further validated using various experiments reported in the following subsequent sections. 


\subsection{Experimentation using Different Fundus Image Databases}

Experimentations are performed for both the proposed algorithms and performance metrics; RMSE, PSNR, SSIM were calculated for all these databases and run time for all the databases explored.

\subsubsection{Experiment 1}

Digital Fundus Images for Vessel Extraction (DRIVE) database consisting of 40 colour fundus images were taken in this experiment. All images are digitized using Cannon CR5 3CCD camera with 45 degrees FOV. Each image is of size $565 \times 584$ with 24 bits resolution per pixel. Ground truth vessel segmentation was given for all the images which were labeled by hand by ophthalmologist [20]. Table 1 shows RMSE, PSNR, SSIM and run time elapsed computed for MBVEKA and BVEFA techniques for ten images.

Table 1. Performance Parameters Comparison for sample images taken from DRIVE database

\begin{tabular}{ccccccccc}
\hline Test & \multicolumn{2}{c}{ RMSE } & \multicolumn{2}{c}{ PSNR (dB) } & \multicolumn{2}{c}{ SSIM } & \multicolumn{2}{c}{ Time Elapsed (sec) } \\
\cline { 2 - 8 } Samples & MBVEKA & BVEFA & MBVEKA & BVEFA & MBVEKA & BVEFA & MBVEKA & BVEFA \\
\hline Img_1 & 12.9746 & 0.4187 & 25.8689 & 55.6929 & 0.7041 & 0.9909 & 2.4984 & 1.7030 \\
Img_2 & 12.9605 & 0.4301 & 25.8783 & 55.4590 & 0.6394 & 0.9924 & 2.4677 & 1.5238 \\
Img_3 & 12.4696 & 0.4063 & 26.2137 & 55.9533 & 0.7672 & 0.9872 & 4.4582 & 1.5815 \\
Img_4 & 13.0382 & 0.4082 & 25.8264 & 55.9114 & 0.5976 & 0.9890 & 3.7574 & 1.6352 \\
Img_5 & 12.8962 & 0.4555 & 25.9215 & 54.9606 & 0.6615 & 0.9948 & 2.6913 & 1.5404 \\
Img_6 & 12.8591 & 0.5046 & 25.9466 & 54.0705 & 0.6962 & 0.9968 & 2.5767 & 1.6095 \\
Img_7 & 12.9701 & 0.4172 & 25.8719 & 55.7228 & 0.6473 & 0.9908 & 2.5267 & 1.6467 \\
Img_8 & 12.0292 & 0.4288 & 25.8324 & 55.4849 & 0.6356 & 0.9931 & 2.5358 & 1.5669 \\
Img_9 & 12.3792 & 0.4786 & 26.2769 & 54.5305 & 0.6664 & 0.9958 & 2.4799 & 1.8224 \\
Img_10 & 12.6150 & 0.5126 & 26.1130 & 53.9345 & 0.6839 & 0.9968 & 2.4968 & 1.5665 \\
\hline
\end{tabular}

From Table 1 it is revealed that RMSE value for MBVEKA and BVEFA lies in the range of 12-13 and 0.4 to 0.5 respectively which consequently provides PSNR ranging from $25 \mathrm{~dB}$ to $26 \mathrm{~dB}$ for MBVEKA and $53 \mathrm{~dB}$ to 55dB for BVEFA. Lower value of RMSE provides higher PSNR range which indicated better image quality. BVEFA yields higher SSIM value as compared to MBVEKA elapsing much lower computational time.

\subsubsection{Experiment 2}

Structured Analysis of Retina (STARE) consists of 20 fundus images captured by TopCon TRV-50 fundus camera with 35 degrees of FOV. Each image is of $605 \times 700$ pixel with 24 bits per pixel resolution. Ground truth vessel segmentation was created for all the twenty images which are labeled manually by the professional expert [21]. The proposed techniques were employed on ten images of this database and results are reported in Table 2 that shows RMSE, PSNR, SSIM and run time elapsed computed for MBVEKA and BVEFA.

Table 2. Performance Parameters Comparison for sample images taken from STARE database

\begin{tabular}{|c|c|c|c|c|c|c|c|c|}
\hline \multirow{2}{*}{$\begin{array}{c}\text { Test } \\
\text { Samples }\end{array}$} & \multicolumn{2}{|c|}{ RMSE } & \multicolumn{2}{|c|}{ PSNR (dB) } & \multicolumn{2}{|c|}{ SSIM } & \multicolumn{2}{|c|}{ Time Elapsed (sec) } \\
\hline & MBVEKA & BVEFA & MBVEKA & BVEFA & MBVEKA & BVEFA & MBVEKA & BVEFA \\
\hline Img_1 & 11.7299 & 0.3880 & 26.7449 & 56.3525 & 0.7006 & 0.9842 & 0.6335 & 1.1559 \\
\hline Img_3 & 12.8966 & 0.3442 & 25.9213 & 57.3936 & 0.7877 & 0.9895 & 0.6160 & 0.3430 \\
\hline Img_4 & 12.1683 & 0.3883 & 26.4262 & 56.3467 & 0.7287 & 0.9836 & 0.5916 & 0.3411 \\
\hline Img_6 & 10.7870 & 0.4091 & 27.4728 & 55.8932 & 0.7042 & 0.9811 & 0.6025 & 0.2911 \\
\hline Img_7 & 11.6832 & 0.4156 & 26.7795 & 55.7553 & 0.6994 & 0.9813 & 0.6333 & 0.3001 \\
\hline Img_8 & 9.3664 & 0.4288 & 28.6993 & 55.4849 & 0.6985 & 0.9796 & 0.6151 & 0.2997 \\
\hline Img_9 & 11.2482 & 0.4600 & 27.1091 & 54.8756 & 0.7734 & 0.9750 & 0.5497 & 0.2979 \\
\hline
\end{tabular}

Table 2 depicts that the performance indices for MBVEKA ranges from 9 to 12 for RMSE, $25 \mathrm{~dB}$ to $29 \mathrm{~dB}$ for PSNR and 0.69 to 0.79 for SSIM value at runtime ranging from 0.5 to 0.7 seconds. Performance achieved for BVEFA provides RMSE ranging from 0.3 to 0.5 , PSNR ranging from $54 \mathrm{~dB}$ to $58 \mathrm{~dB}$, SSIM ranging from 0.97 to 0.99 at runtime of 0.2 to 0.4 seconds. 


\subsubsection{Experiment 3}

DIARETDB1 database consists of 89 colour images out of which 84 images contains the sign of nonproliferative DR and remainaing 5 are normal fundus images containg no sign of DR. All the images were captured with a digital fundus camera having 50 degree FOV with varying imaging controlled system in Kuopio University Hospital, Finland. These fundus images were inspected to find hard exudates, hemorrhages and microaneurysms using specialized softwares [22]. The proposed techniques were employed on ten images of this database and results are reported in Table 3.

Table 3. Performance Parameters Comparison for sample images taken from DIARETDB1 database

\begin{tabular}{ccccccccc}
\hline Test & \multicolumn{2}{c}{ RMSE } & \multicolumn{2}{c}{ PSNR $(\mathrm{dB})$} & \multicolumn{2}{c}{ SSIM } & \multicolumn{2}{c}{ Time Elapsed (sec) } \\
\cline { 2 - 8 } Samples & MBVEKA & BVEFA & MBVEKA & BVEFA & MBVEKA & BVEFA & MBVEKA & BVEFA \\
\hline Img_1 & 11.7476 & 0.7321 & 26.7318 & 50.8383 & 0.7525 & 0.9598 & 10.9177 & 6.5241 \\
Img_2 & 11.8566 & 0.7429 & 26.6516 & 50.7114 & 0.7417 & 0.9573 & 10.5504 & 6.4466 \\
Img_3 & 10.9724 & 0.5449 & 27.3247 & 53.4032 & 0.7236 & 0.9583 & 11.1990 & 6.4577 \\
Img_4 & 11.8267 & 0.5628 & 26.6735 & 53.1229 & 0.6910 & 0.9555 & 10.7314 & 6.4522 \\
Img_5 & 11.9838 & 0.4597 & 26.5588 & 54.8797 & 0.7127 & 0.9708 & 10.9975 & 6.4159 \\
Img_6 & 12.2776 & 0.4817 & 26.3485 & 54.4740 & 0.7848 & 0.9678 & 10.8889 & 6.4911 \\
Img_7 & 9.9016 & 0.6006 & 28.2166 & 52.5581 & 0.6620 & 0.9491 & 11.5345 & 6.5361 \\
Img_8 & 11.0865 & 0.5056 & 27.2348 & 54.5349 & 0.7637 & 0.9643 & 10.8086 & 6.3963 \\
Img_9 & 10.5695 & 0.5771 & 27.6497 & 52.9050 & 0.6665 & 0.9531 & 10.8662 & 6.4139 \\
Img_10 & 11.7963 & 0.5223 & 26.6959 & 53.7723 & 0.7467 & 0.9619 & 10.9774 & 6.3603 \\
\hline
\end{tabular}

Tabular representation of performance indices DIARETDB1 dataset reveals: RMSE, 9 to12; PSNR, $26 \mathrm{~dB}$ to $28 \mathrm{~dB}$; SSIM, 0.66 to 0.78 and time elapsed, 10 to 12 seconds for MBVEKA.-RMSE, 0.4 to 0.8 ; PSNR, $50 \mathrm{~dB}$ to $55 \mathrm{~dB}$ and SSIM, 0.95 to 0.97 is obtained for BVEFA at comparatively less computation time of 6 to 7 seconds.

\subsubsection{Experiment 4}

Method of Evaluating Segmentation and Indexing Techniques in the Field of Retianal Opthalmology (MESSIDOR) consists of 1200 eye fundus color images, out of which 800 images are acquired with pupil dilation and 400 without dilation. These images were acquired by 3 ophthalmologic departments using $3 \mathrm{CCD}$ camera on a Topcon TRC NW6 non-mydriatic retinography with 45 degrees FOV, captured using 8 bits per color plane at $1440 \times 960,2240 \times 1488$ or $2304 \times 1536$ pixels [23]. The proposed techniques were employed on ten images of this database and results are reported in Table 4.

Table 4. Performance Parameters Comparison for sample images taken from MESSIDOR database

\begin{tabular}{|c|c|c|c|c|c|c|c|c|}
\hline \multirow{2}{*}{$\begin{array}{c}\text { Test } \\
\text { Samples }\end{array}$} & \multicolumn{2}{|c|}{ RMSE } & \multicolumn{2}{|c|}{ PSNR (dB) } & \multicolumn{2}{|c|}{ SSIM } & \multicolumn{2}{|c|}{ Time Elapsed (sec) } \\
\hline & MBVEKA & BVEFA & MBVEKA & BVEFA & MBVEKA & BVEFA & MBVEKA & BVEFA \\
\hline Img_1 & 2.4993 & 0.4039 & 40.1743 & 56.0115 & 0.7005 & 0.9788 & 0.1299 & 0.3857 \\
\hline Img_2 & 2.6632 & 0.3254 & 39.6228 & 57.8818 & 0.7284 & 0.9875 & 0.6535 & 0.3586 \\
\hline Img_3 & 4.2890 & 0.4426 & 35.4836 & 55.2104 & 0.7444 & 0.9792 & 0.5335 & 0.2922 \\
\hline Img_4 & 4.5341 & 0.4659 & 35.0011 & 54.7642 & 0.7637 & 0.9756 & 0.5152 & 0.2797 \\
\hline Img_5 & 4.3329 & 0.3933 & 35.3952 & 56.2359 & 0.6985 & 0.9856 & 0.5142 & 0.3044 \\
\hline Img_6 & 4.5098 & 0.4967 & 35.0475 & 54.2073 & 0.7568 & 0.9671 & 0.5202 & 0.2706 \\
\hline Img_7 & 4.7283 & 0.3778 & 34.6367 & 56.5835 & 0.7996 & 0.9861 & 0.5083 & 0.2504 \\
\hline Img_8 & 3.5238 & 0.4987 & 37.1904 & 54.1722 & 0.7655 & 0.9706 & 0.5355 & 0.2859 \\
\hline Img_9 & 2.7716 & 0.4560 & 39.2759 & 54.9501 & 0.6999 & 0.9736 & 0.5178 & 0.2890 \\
\hline Img_10 & 3.0864 & 0.4325 & 38.3415 & 55.4100 & 0.7552 & 0.9800 & 0.5194 & 0.2752 \\
\hline
\end{tabular}

It is seen in Table 4 that MESSIDOR dataset provides PSNR ranging from $54 \mathrm{~dB}$ to $58 \mathrm{~dB}$ for BVEFA method and $35 \mathrm{~dB}$ to $40 \mathrm{~dB}$ for MBVEKA method. SSIM index ranges from 0.69 to 0.79 for MBVEKA method and higher values are observed for BVEFA ranging from 0.96 to 0.99. Less time is elapsed for BVEFA method making the approach time efficient.

Tabular comparison of MBVEKA and BVEFA reveals that BVEFA gives low value of RMSE nearly approaching towards zero which in turn gives better PSNR value ranging from $50 \mathrm{~dB}$ to $58 \mathrm{~dB}$ which is 
comparatively better than MBVEKA approach. It is seen that SSIM value obtained from BVEFA approach is strongly approaching towards 1 and best results of SSIM can be seen for DRIVE database images. Although these results vary from image to image but on comparing both the approaches, BVEFA is proven better than MBVEKA approach. Runtime comparison of images taken from different databases using MBVEKA and BVEFA approaches is also done. It is observed that the maximum runtime is shown by the images of DIARETDB1 database because these images have highest FOV of 50 degrees which makes these images takes more time to process as they have higher resolution as compared to images obtained from all other databases.

\subsubsection{Experiment 5}

This experiment is done using DRIVE [20] database because the ground truth blood vessels are only available for DRIVE database, therefore the statistical performance metrics Jaccard's index, Dice Coefficient, Sensitivity, Specificity and Accuracy are calculated for DRIVE database images. Tabular comparison of these parameters is shown in table 5 and table 6 for MBVEKA and BVEFA approaches respectively.

Table 5. Comparison for DRIVE Database images for MBVEKA Algorithm

\begin{tabular}{|c|c|c|c|c|c|c|}
\hline $\begin{array}{c}\text { DRIVE } \\
\text { database } \\
\text { Images }\end{array}$ & $\begin{array}{l}\text { Jaccard's } \\
\text { Index }\end{array}$ & $\begin{array}{c}\text { Dice } \\
\text { Coefficient }\end{array}$ & SSIM & Sensitivity & Specificity & Accuracy \\
\hline Img_1 & 0.3042 & 0.4665 & 0.7041 & 0.5640 & 0.9310 & 0.9036 \\
\hline Img_2 & 0.3609 & 0.5304 & 0.6394 & 0.6365 & 0.9242 & 0.8982 \\
\hline Img_3 & 0.2459 & 0.3947 & 0.7672 & 0.7265 & 0.8623 & 0.8533 \\
\hline Img_4 & 0.3767 & 0.5473 & 0.5976 & 0.5829 & 0.9283 & 0.8883 \\
\hline Img_5 & 0.2720 & 0.4277 & 0.6615 & 0.4042 & 0.9484 & 0.8962 \\
\hline Img_6 & 0.3036 & 0.4658 & 0.6962 & 0.5826 & 0.9162 & 0.8883 \\
\hline Img_7 & 0.3460 & 0.5141 & 0.6473 & 0.6104 & 0.9261 & 0.8983 \\
\hline Img_8 & 0.3589 & 0.5282 & 0.6356 & 0.6108 & 0.9240 & 0.8934 \\
\hline Img_9 & 0.3123 & 0.4759 & 0.6664 & 0.5633 & 0.9262 & 0.8957 \\
\hline Img_10 & 0.3125 & 0.4762 & 0.6839 & 0.6029 & 0.9209 & 0.8959 \\
\hline Average & 0.3193 & 0.4827 & 0.6699 & 0.5884 & 0.9207 & 0.8911 \\
\hline
\end{tabular}

Table 6. Comparison for DRIVE Database images for BVEFA Algorithm

\begin{tabular}{ccccccc}
\hline $\begin{array}{c}\text { DRIVE } \\
\text { database } \\
\text { Images }\end{array}$ & $\begin{array}{c}\text { Jaccard's } \\
\text { Index }\end{array}$ & $\begin{array}{c}\text { Dice } \\
\text { Coefficient }\end{array}$ & SSIM & Sensitivity & Specificity & Accuracy \\
\hline Img_1 & 0.9851 & 0.9925 & 0.9909 & 1.0000 & 0.9815 & 0.9851 \\
Img_2 & 0.9848 & 0.9924 & 0.9924 & 1.0000 & 0.9884 & 0.9848 \\
Img_3 & 0.9849 & 0.9924 & 0.9872 & 1.0000 & 0.9894 & 0.9849 \\
Img_4 & 0.9860 & 0.9930 & 0.9890 & 1.0000 & 0.9806 & 0.9860 \\
Img_5 & 0.9859 & 0.9929 & 0.9948 & 1.0000 & 0.9895 & 0.9859 \\
Img_6 & 0.9855 & 0.9927 & 0.9968 & 1.0000 & 0.9855 & 0.9855 \\
Img_7 & 0.9880 & 0.9940 & 0.9908 & 1.0000 & 0.9808 & 0.9880 \\
Img_8 & 0.9883 & 0.9941 & 0.9931 & 1.0000 & 0.9838 & 0.9883 \\
Img_9 & 0.9856 & 0.9927 & 0.9958 & 1.0000 & 0.9865 & 0.9856 \\
Img_10 & 0.9849 & 0.9924 & 0.9968 & 1.0000 & 0.9849 & 0.9849 \\
\hline Average & 0.9859 & 0.9929 & 0.9928 & 1.0000 & 0.9851 & 0.9859 \\
\hline
\end{tabular}

Tables 5 and 6 demonstrate the variation in statistical parameters: Jaccard's index, Dice Coefficient, Sensitivity, Specificity and Accuracy of blood vessel extractions. We observed from table 6 that with the DRIVE dataset, proposed BVEFA achieved highest accuracy of $98 \%$. Table 5 shows different parameters; Jaccard's index, Dice Coefficient, Sensitivity, Specificity and Accuracy for 10 images from DRIVE database and the average accuracy obtained for MBVEKA approach is $89 \%$. This comparison shows that BVEFA approach yield better results in terms of PSNR, SSIM, Jaccard's index, Dice Coefficient, Sensitivity, Specificity and Accuracy.

A comparison chart showing different research initiatives for blood vessel extraction is depicted in table 7 along with the image processing techniques employed and performance metrics evaluated. 
Table 7. Comparison of different Optical Disc Removal and Retinal Blood Vessel Extraction Techniques

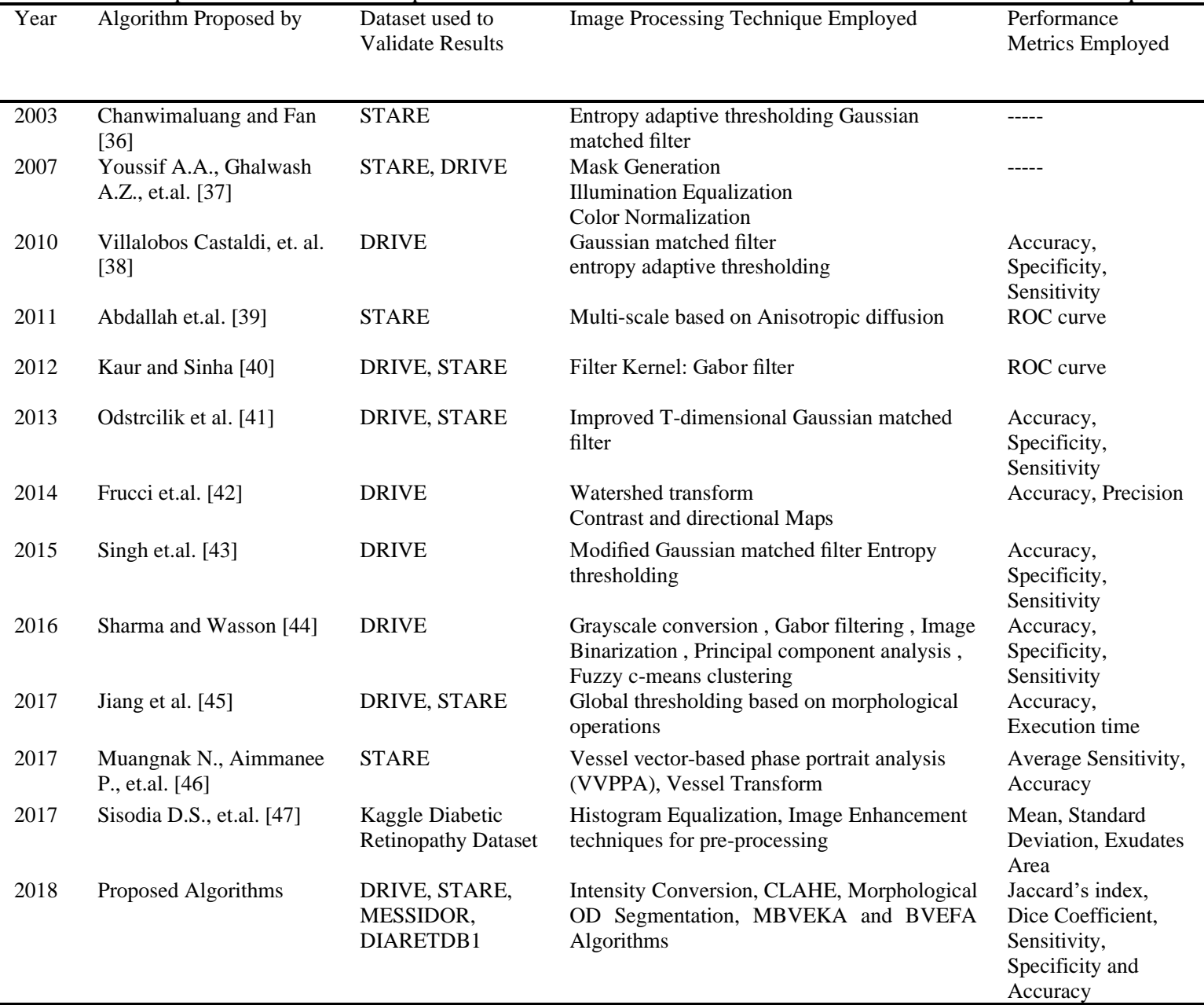

Upon comparing different blood vessel extraction techniques from the year 2003 to 2017 it was found that most of the analysis was done taking two main databases, DRIVE and STARE database but our proposed techniques are tested for 4 different databases and it provides satisfactory results. Many other performance metrics are exploited for our research analysis unlike other blood vessel extraction methods reported in the literature. A tabular comparison for different techniques reported in the previous literatures is made in table 8 comparing the average accuracies of the proposed technique with the already employed blood vessel extraction techniques.

Table 8. Comparative Analysis of Proposed Blood Vessel Extraction Technique with the Existing Techniques

\begin{tabular}{ccc}
\hline S. No. & Employed Algorithms & Accuracy \\
\hline 1. & Ana Salazar-Gonzalez, et.al. [17] & $94 \%$ \\
2. & Sharma and Wasson [44] & $95 \%$ \\
3. & Jiang et al. [45] & $95.88 \%$ \\
4. & Jyotiprava Dash and Nilamani Bhoi [48] & $95 \%$ \\
5. & Proposed Technique [BVEFA] & $98 \%$ \\
\hline
\end{tabular}

The proposed method BVEFA provides the best average accuracy of $98 \%$ as compared to the already existing blood vessel extraction techniques and it is indicated in Table 8 . The advantage of the proposed method is its ease of implementation as well as robustness as compared to the other existing methods. A small drawback of the proposed method lies in case of pathological fundus images containing some lesions as in this case the system may lead to inaccurate segmentation and blood vessel extraction. However, this drawback can be resolved in the future research work by employing deep learning methods to facilitate screening of diabetic retinopathy and other eye related complications. 


\section{CONCLUSION}

For complete assessment of the proposed technique, we have performed five sets of experimental evaluations. In the first four experiments, the performance metrics of the proposed technique are analyzed for all the four datasets. In the fifth experiment, the performance of blood vessel extraction is analyzed and compared with gold standard available for DRIVE database to assess the proposed vessel extraction for retinal abnormality detection. This paper presents an extensive analysis of two proposed blood vessel extraction techniques; BVEFA not only achieves better results as compared to MBVEKA approach giving average accuracy of $98 \%$ but it also ensures extraction of fine vessel branches. The proposed method outperforms for DRIVE databases than most existing methods and STARE database in terms of image quality parameters. The algorithms presented here are both robust to variations in image and computationally less complex when compared to existing algorithms. Blood vessel extraction using BVEFA can facilitate the ophthalmologist for diagnosis and screening of diabetes retinopathy symptoms for early stage detection. These techniques are employed to enhance the fundus images which can be used for automated detection of signs indicating DR.

\section{REFERENCES}

[1] Shivaram J.M., Patil R., Aravind H. Automated Detection and Quantification of Haemorrhages in Diabetic Retinopathy Images Using Image Arithmetic and Mathematical Morphology Methods. International Journal of Recent Trends in Engineering. 2009; 2(6): 174-176.

[2] World Health Organization. Definition, diagnosis and classification of diabetes mellitus and its complications, Part 1, Diagnosis and classification of diabetes mellitus. Tech. rep., World Health Organization Non communicable Geneva, 1999.

[3] World Health Organization and the International Diabetes Federation. Diabetes action now, An initiative of world health organization and the international diabetes federation, 2004.

[4] Gharaibeh N. Y. A Novel Approach for Detection of Microaneurysms in Diabetic Retinopathy from Fundus Images. Computer and Information Science. 2017; 10(1): 1-15.

[5] Sudeshna Sil Kar, Santi P. Maity. Automatic Detection of Retinal Lesions for Screening of Diabetic Retinopathy. IEEE Transactions on Biomedical Engineering. 2016; 1-9.

[6] Keerthi Ram, Gopal Datt Joshi, Jayanthi Sivaswamy. A Successive Clutter-Rejection-Based Approach for Early Detection of Diabetic Retinopathy. IEEE Transactions on Biomedical Engineering. 2011; 58(3): 664-673.

[7] Lama Seoud, Thomas Hurtut, Jihed Chelbi, Farida Cheriet, J.M. Pierre Langlois. Red Lesion Detection Using Dynamic Shape Features for Diabetic Retinopathy Screening. IEEE Transactions on Medical Imaging. 2016; 35(4): 1116- 1126.

[8] Istvan Lazar, Andras Hajdu. Retinal Microaneurysm Detection Through Local Rotating Cross-Section Profile Analysis. IEEE Transactions on Medical Imaging. 2013; 32(2): 400-407.

[9] Arturo Aquino, Manuel Emilio Gegúndez-Arias, and Diego Marín. Detecting the Optic Disc Boundary in Digital Fundus Images Using Morphological, Edge Detection, and Feature Extraction Techniques. IEEE Transactions on Medical Imaging. 2011; 29(11): 1860- 1869.

[10] Rahim S.S., Palade V., et.al. Detection of Diabetic Retinopathy and Maculopathy using Fuzzy Image Processing. Springer. 2015: 379-388.

[11] Sunita Sarangi, Arpita Mohapatra, Sukant Kumar Sabut. A comparative evaluation of diabetic retinal vascular structures using edge detection techniques. International Journal Telemedicine and Clinical Practices. 2015; 1(1): 111-120.

[12] Sisodia D.S., Nair S., Khobragade P. Diabetic Retinal Fundus Images, Preprocessing and Feature Extraction for Early Detection of Diabetic Retinopathy. Biomedical \& Pharmacology Journal. 2017; 10(2): 615-626.

[13] Rahim S.S., et.al., Automatic Screening and Classification of Diabetic Retinopathy using Fuzzy Image Processing. Springer. 2016.

[14] Gao Weiwei, Zuo Jing. Automated Detection of Hard Exudates in Fundus Images using Improved OTSU Thresholding and SVM. International Journal of Computer Science \& Engineering Survey (IJCSES). 2016; 6(1): 1-11.

[15] Partovi M., Rasta S.H., Javadzadeh A. Automatic Detection of Retinal Exudates in fundus images of Diabetic Retinopathy Patient. Journal of Analytical Research in Clinical Medicine. 2016; 4(2): 104-109.

[16] Majumdar J., et. al. An Automated Graphical User Interface based System for the Extraction of Retinal Blood Vessels using Kirsch's Template. International Journal of Advanced Computer Science and Application (IJACSA). 2015; 6(6): 86-93.

[17] Ana Salazar-Gonzalez, Djibril Kaba, Yongmin Li, and Xiaohui Liu. Segmentation of the Blood Vessels and Optic Disk in Retinal Images. IEEE Journal of Biomedical and Health Informatics. 2014; 18(6). 
[18] Thomas N., Mahesh T.Y. Detecting Clinical Features of Diabetic Retinopathy. IJERT. 2014; 3(8): 558561.

[19] Khan M.W., Diabetic Retinopathy Detection using Image Processing, A Survey. IJETR. 2013: 16-20.

[20] Staal J. J., Abramoff M. D., Niemeijer M., Viergever M. A., Ginneken B. V. Ridge based vessel segmentation in color images of the retina. IEEE Transaction on Medical Imaging,2004; 23: 501-509.

[21] Hoover A., Kouznetsova V., Goldbaum M. Locating blood vessels in retinal images by piecewise threshold probing of a matched filter response. IEEE Transactions on Medical Imaging. 2000; 19(3): 203-210.

[22] Kauppi T., Kalesnykiene V., KamarainenJ., et al. The DIARETDB1 diabetic retinopathy database and evaluation protocol. in Proceedings of the British Machine Vision Conference (BMVC '07), 2007: 1-10, Warwick, UK, September.

[23] MESSIDOR, Methods for Evaluating Segmentation and Indexing technique Dedicated to Retinal Ophthalmology, http,//messidor.crihan.fr/index-en.php, 2004.

[24] Dogra J., Sood M., Jain S., Prashar N. Segmentation of Magnetic Resonance Images of Brain using Thresholding Techniques. Proceedings of the IEEE International Conference on signal processing and control (ISPCC 2017). 2017: 311-315.

[25] Bhusri S., Jain S., Virmani J. Breast Lesions Classification using the Amalagation of morphological and texture features. International Journal of Pharma and Bio Sciences. 2016; 7(2): 617-624.

[26] Sharma S., Jain S., Bhusri S. Two Class Classification of Breast Lesions using Statistical and Transform Domain features. Journal of Global Pharma Technology. 2017; 9(7): 18-24.

[27] Sisodia D.S., Nair S., Khobragada P. Diabetic Retinal Fundus Images, Preprocessing and Feature Extraction For Early Detection of Diabetic Retinopathy. Biomedical \& Pharmacology Journal. 2017; 10(2): 615-626.

[28] R.C. Gonzalez and R.E. Woods. Digital Image processing (2nd edition), Prentice-Hall, Englewood Cliffs, NJ. 2002.

[29] Bethanney Janney J., Sindu Divakaran, Sheeba Abraham, Meera.G, Uma Shankar.G. Detection and classification of exudates in retinal image using image processing techniques. Journal of Chemical and Pharmaceutical Science. 2015; 8(3): 541-546.

[30] Rajaiah R.P., Britto R.J. Optic Disc Boundary Detection and Cup Segmentation for Prediction of Glaucoma. International Journal of Science, Engineering and Technology Research (IJSETR). 2014; 3(10).

[31] Shahriar Badsha, Ahmed Wasif Reza, Kim Geok Tan, Kaharudin Dimyati. A New blood Vessel Extraction Technique using Edge Enhancement and Object Classification. Journal of Digital Imaging. 2013; 26(6): 1107-1115.

[32] Becerikli, Yasar, Tayfun M. Karan. A new fuzzy approach for edge detection. International WorkConference on Artificial Neural Networks. Springer, Berlin, Heidelberg (2005).

[33] Sood M. Performance Analysis of Classifiers for Seizure Diagnosis for Single Channel EEG Data. Biomed Pharmacol Journal. 2017; 10(2): 795-803.

[34] Bhardwaj C., Urvashi, Sood M. Implementation and Performance Assessment of Compressed Sensing for Images and Video Signals. Journal of Global Pharma Technology. 2017; 6(9): 123-133.

[35] Urvashi, Bhardwaj C. Sood M. Effectiveness of Reconstruction Methods in Compressive Sensing for Biomedical Images. Journal of Global Pharma Technology. 2017; 6(9): 134-143.

[36] ChanwimaluangT., Fan G. An efficient algorithm for extraction of anatomical structures in retinal images. In Proceedings of the 2003 International Conference on Image Processing (Cat.No.03CH37429), Barcelona, Spain.1091. 2003: 1093-1096.

[37] Youssif A.A., Ghalwash A.Z., Ghoneim A.S. A comparative evaluation of preprocessing methods for automatic detection of retinal anatomy. in Proceedings of the Fifth International Conference on Informatics and Systems (INFOS 07). (2430) 2007: 24-30.

[38] Villalobos-Castaldi F.M., Felipe-Riverón E.M., Sánchez-Fernández L.P. A fast, efficient and automated method to extract vessels from fundus image. Journal of Vision.13. 2010: 263-270.

[39] Abdallah M.B., Malek J., Krissian K., Tourki R. An automated vessel segmentation of retinal images using multiscalevesselness. In Proceedings of the Eighth International Multi-Conference on Systems, Signals \& Devices, Sousse, Tunisia.2011:1-6.

[40] Kaur J., Sinha H. Automated detection of retinal blood vessels in diabetic retinopathy using Gabor filter.Int. J. Comput. Sci. Netw. Secur.2012; 109(12).

[41] Odstrcilik J., Kolar R., BudaiA., et.al. Retinal vessel segmentation by improved matched filtering, Evaluation on a new high-resolution fundus image database. IET Image Processing. 2013; 7: 373-383. 
[42] Frucci M., Riccio D., Baja G.S.D., Serino L. Using Contrast and Directional Information for Retinal Vessels Segmentation, In Proceedings of the 2014 Tenth International Conference on Signal-Image Technology and Internet-Based Systems, Marrakech, Morocco. 2014: 592-597.

[43] Singh N.P., Kumar R., Srivastava R. Local entropy thresholding based fast retinal vessels segmentation by modifying matched filter. In Proceedings of the International Conference on Computing, Communication \&Automation, Noida, India, 2015: 1166-1170.

[44] Sharma S., Wasson E.V. Retinal Blood Vessel Segmentation Using Fuzzy Logic. Journal of Network Communications and Emerging Technologies. 2015; 4.

[45] Jiang Z., Yepez J., An S., Ko S. Fast, accurate and robust retinal vessel segmentation system. Biocybernetics and Biomedical Engineering Journal. 2017; 37: 412-421.

[46] Muangnak N., AimmaneeP., Makhanov S. Automatic optic disc detection in retinal images using hybrid vessel phase portrait analysis. International Federation for Medical and Biological Engineering. 2017.

[47] Sisodia D.S., Nair S., Khobragade P. Diabetic Retinal Fundus Images, Preprocessing and Feature Extraction for Early Detection of Diabetic Retinopathy. Biomedical \& Pharmacology Journal. 2017; 10(2): 615-626.

[48] Dash J., Bhoi N. An Unsupervised Approach for Extraction of Blood Vessels from Fundus Images. Journal of Digital Imaging. 2018. 